\title{
User perspectives in public transport timetable optimisation
}

\author{
Jensen, Jens Parbo; Nielsen, Otto Anker; Prato, Carlo Giacomo
}

Published in:

Transportation Research. Part C: Emerging Technologies

Link to article, DOI:

10.1016/j.trc.2014.09.005

Publication date:

2014

Document Version

Publisher's PDF, also known as Version of record

Link back to DTU Orbit

Citation $(A P A)$ :

Jensen, J. P., Nielsen, O. A., \& Prato, C. G. (2014). User perspectives in public transport timetable optimisation. Transportation Research. Part C: Emerging Technologies, 48, 269-284. https://doi.org/10.1016/j.trc.2014.09.005

\section{General rights}

Copyright and moral rights for the publications made accessible in the public portal are retained by the authors and/or other copyright owners and it is a condition of accessing publications that users recognise and abide by the legal requirements associated with these rights.

- Users may download and print one copy of any publication from the public portal for the purpose of private study or research.

- You may not further distribute the material or use it for any profit-making activity or commercial gain

- You may freely distribute the URL identifying the publication in the public portal 


\title{
User perspectives in public transport timetable optimisation
}

\author{
Jens Parbo*, Otto Anker Nielsen, Carlo Giacomo Prato \\ Technical University of Denmark, Department of Transport, Bygningstorvet 116B, 2800 Kgs. Lyngby, Denmark
}

\section{A R T I C L E I N F O}

\section{Article history:}

Received 12 March 2014

Received in revised form 10 September

2014

Accepted 10 September 2014

\section{Keywords:}

Bus timetabling

Public transport optimisation

Passenger behaviour

Waiting time

Large-scale application

\begin{abstract}
A B S T R A C T
The present paper deals with timetable optimisation from the perspective of minimising the waiting time experienced by passengers when transferring either to or from a bus. Due to its inherent complexity, this bi-level minimisation problem is extremely difficult to solve mathematically, since timetable optimisation is a non-linear non-convex mixed integer problem, with passenger flows defined by the route choice model, whereas the route choice model is a non-linear non-continuous mapping of the timetable. Therefore, a heuristic solution approach is developed in this paper, based on the idea of varying and optimising the offset of the bus lines. Varying the offset for a bus line impacts the waiting time passengers experience at any transfer stop on the bus line.

In the bi-level timetable optimisation problem, the lower level is a transit assignment calculation yielding passengers' route choice. This is used as weight when minimising waiting time by applying a Tabu Search algorithm to adapt the offset values for bus lines. The updated timetable then serves as input in the following transit assignment calculation. The process continues until convergence.

The heuristic solution approach was applied on the large-scale public transport network in Denmark. The timetable optimisation approach yielded a yearly reduction in weighted waiting time equivalent to approximately 45 million Danish kroner ( 9 million USD).
\end{abstract}

(c) 2014 Elsevier Ltd. All rights reserved.

\section{Introduction}

In a report from the Capital Region of Denmark (RH, 2009), it was estimated that 11.5 billion Danish kroner (DKK) will be lost due to travellers being delayed because of congestion in the Copenhagen Region in 2015. Furthermore, it was stated in the report that, to avoid the outlined scenario, people ought to start travelling by public transport rather than by car. The question is how this change in the market share between private and public transport is actually realised?

The present paper deals with timetable optimisation from the perspective of minimising the waiting time experienced when transferring either to or from a bus.

\subsection{Literature review}

Designing an attractive transit network is an important and strategic task, in the literature often referred to as the Transit Route Network Design Problem (TRNDP). Based on an existing bus network, Bielli et al. (2002) aimed at improving the performance and reducing the need for rolling stock by adapting lines and their frequency. Lee and Vuchic (2005) tried to design an optimal transit network as a compromise between minimal travel time, transit operator's profit maximisation and

\footnotetext{
* Corresponding author. Tel.: +45 45256532.

E-mail addresses: jepar@transport.dtu.dk (J. Parbo), oan@transport.dtu.dk (O.A. Nielsen), cgp@transport.dtu.dk (C.G. Prato).
} 
minimisation of social costs. Elaborating mainly on the travel time description, Fan and Machemehl (2006) considered the transit route network design problem but separated travel time into four components (walking time, waiting time, in-vehicle time and transfer cost). The TRNDP has received much attention in the literature, and its significant contribution was notably summarised in two reviews by Kepaptsoglou and Karlaftis (2009), focusing on design objectives, operating environments, and solution approaches, and Guihaire and Hao (2008), focusing on unifying the area. Regarding future developments within this area, Kepaptsoglou and Karlaftis (2009) recommended that the focus should be on transfer policies and passenger transfer related items as waiting and walking distances, while Guihaire and Hao (2008) suggested that the focus should be on privatisation and deregulation, as well as integration and intermodality among transit networks by focusing on improving transfers globally instead of looking at within-mode transfers.

In the literature, several solutions have been proposed to the timetable optimisation problem with various approaches to the consideration of transfers. One of the problems that have received much attention is the Timetable Synchronisation Problem (TTSP, e.g., Ceder, 2007; Liu et al., 2007; Ibarra-Rojas and Rios-Solis, 2012), which aims at maximising the number of simultaneous arrivals at transfer stations. Wong et al. (2008) developed a timetable optimisation model trying to minimise the total passenger transfer waiting times by changing the offset of the bus lines. This approach was also used, though with different objectives, by Bookbinder and Désilets (1992), Knoppers and Muller (1995), Cevallos and Zhao (2006), Hadas and Ceder (2010) and Petersen et al. (2012). Guihaire and Hao (2010) maximised the quality and quantity of transfer opportunities. While the quantity was self-explanatory, the quality was a twofold concept: firstly, it was based on the number of passengers; secondly, it was based on an ideal transfer time, i.e., a cost function was introduced to force the transfer time to be as close as possible to the ideal one. Niu and Zhou (2013) applied a timetable optimisation approach taking into account the passengers boarding at crowded stations. The objective was to minimise passengers' waiting time at stops and also reduce the waiting time passengers who were not able to board their desired service suffered because of congestions. They applied a genetic algorithm to solve the problem for each station in a double-track corridor. De Palma and Lindsey (2001) tried to minimise schedule delay (i.e., difference between preferred and actual departure time) by choosing the best timetable among a finite set of a priori created timetables. Taking a more holistic and strategic view of the transit network, Zhao and Ubaka (2004) applied two different algorithms to find the optimal set of transit routes to maximise route directness, minimise number of transfers and maximise service coverage. Another alternative perspective was used in the study by Yan et al. (2012), where the objective was to design a reliable bus schedule for fixed bus routes with a series of control points, and the punctuality of the busses was continuously controlled for and it was intended to improve it by letting the drivers recover the schedule by speeding up in order to reach the next control stop on time. Including the requirements for different types of rolling stock, Ceder (2011) developed an extended version of the deficit function to efficiently allocate different types of rolling stock where needed to accommodate the demand on each transit line based on an existing timetable.

In all of these studies, some prior information on users' travel behaviour was used, but passengers were assumed not to change their route choice when the timetable was changed. Normally, one would expect demand to change accordingly, when the supply is changed. In this context, the supply should be seen as the transit system, hence also the timetable, while the demand reflects the transport, namely the passengers' route choice. With this in mind, it seems appropriate to look at some of the timetable optimisation approaches which have considered the balance between supply and demand. Actually, this balance was noted as missing by Zhao and Ubaka (2004) and more recently by Ibarra-Rojas and Rios-Solis (2012). An early study formulated the timetable optimisation problem as a bi-level nonlinear non-convex mixed integer programming problem (Constantin and Florian, 1995). The objective of the upper level was to minimise the total expected travel time plus the waiting time. This was done by changing frequency settings in the timetable. The lower level problem was a transit assignment model with frequencies determined by the upper level. Wang and Lin (2010) developed a bi-level model to minimise operating cost related to the size of the fleet plus the total travel cost for passengers. Here the upper level referred to the determination of service routes and the associated headways. The lower level referred to the route choice behaviour, which was found by using a deterministic Frank-Wolfe loading approach. Ma (2011) applied a bi-level approach for the optimal line frequencies in a transit network, meaning the frequencies that minimise passengers travel time plus the operating cost. The lower level problem (route choice) was solved by using a Cross Entropy Learning algorithm, which was able to find the user equilibrium in transport networks. The upper level problem (optimising line frequencies) used the Hooke-Jeeves algorithm to find improvements in the current solution. Considering the same problem of finding the optimal frequency for a bus network, Yu et al. (2009) applied a bi-level programming model with the objective to reduce passengers' total travel time. In this approach, the upper level determined the bus frequencies by a genetic algorithm while the lower level assigned transit trips to the bus route network by use of a label-marking method. The two levels were solved sequentially until convergence.

One of the first studies to consider transfer time minimisation and also how passengers adjusted their travel patterns accordingly was Feil (2005), who applied a Steepest Descent approach to find the most promising offset changes and evaluated their actual impact with a public assignment model.

\subsection{Objective and contribution}

In the present paper, the objective was to minimise the weighted transfer waiting time. A weight reflecting the number of passengers transferring and their actual value of time was assigned to every transfer. The weight was based on the individual 
passenger's trip purpose. The waiting time was the time that elapsed between the alighting of one run and the boarding of the next run.

The optimisation was performed with the view of finding the optimal departure time (offset) for each bus line to reduce passengers' waiting time when transferring. Instead of treating passengers' route choice as static and predetermined, the approach developed in this paper treated their route choice as pseudo-dynamic. This was done in an iterative process where the output of the timetable optimisation (i.e., the new timetable) served as input to the public assignment model. The output from this public assignment model (i.e., passengers' travel patterns) was then used as input in the timetable. Due to its inherent complexity, finding the optimal offset with this particular objective for the bus lines was extremely difficult analytically. Tabu Search was chosen because of its ability to avoid being trapped in local minima, and also because it had proven to be superior compared to other metaheuristics when bus timetables in Copenhagen were to be optimised (Jansen et al., 2002).

The heuristic solution approach was applied to the highly complex bus network in Denmark. Applying the heuristic solution approach to the large-scale public transport network in Denmark should preferably reduce the waiting time passengers experience when transferring, while keeping their in-vehicle time and their total generalised travel cost at a constant level.

\section{Method}

The current study applied a bi-level timetable optimisation approach, where the objective was to minimise the weighted transfer waiting time. Timetable optimisation (upper level) was integrated with a public assignment model (lower level) to assess how travellers change their behaviour according to the changes imposed in the timetable. This is not a constructive heuristic. Therefore, to make the developed approach work properly, it is necessary to have an existing transit network as an initial solution and being able to run a schedule-based transit assignment, i.e., having a timetable explicitly stating when every transit vehicle departs from its initial stop and when it arrives and departs from the sub sequent stops all the way to its destination.

\subsection{Analytical formulation}

The mathematical formulation of the timetable optimisation problem was as follows:

$$
\min . \sum_{i=1}^{M} \sum_{j=1}^{M} \sum_{s=1}^{N} \omega_{i j}^{S} w_{i j}^{s}
$$

where

$$
\begin{aligned}
& w_{i j}^{s}=\min \left\{\pi_{j}+\alpha_{j}^{s}+\beta_{j}^{s}-\left(\pi_{i}+\alpha_{i}^{s}+\delta^{s}\right) \mid \pi_{j}+\alpha_{j}^{s}+\beta_{j}^{s} \geqslant \pi_{i}+\alpha_{i}^{s}+\delta^{s}\right\} \\
& \omega_{i j}^{s}=\sum_{c} T_{i j c}^{s} \cdot V o T_{c}
\end{aligned}
$$

subject to

$$
\begin{aligned}
& \alpha_{i-1}^{s}+H_{k, i-1, i} \leqslant \alpha_{i}^{s}, \forall k, i, s \in K, M, N \\
& \pi_{k} \geqslant 0, \forall k \in K \\
& H_{k, i-1, i} \geqslant 0, \forall k, i \in K, M \\
& \alpha_{i}^{s} \geqslant 0, \forall i, s \in M, N \\
& \beta_{i}^{s} \geqslant 0, \forall i, s \in M, N \\
& \delta^{s} \geqslant 0, \forall s \in N
\end{aligned}
$$

In the objective function, $w_{i j}^{S}$ is the waiting time between busses $i$ and $j$ at a stop $s, \omega_{i j}^{S}$ is a weight reflecting the importance of every single transfer, $T_{i j c}^{S}$ is the number of passengers transferring between busses $i$ and $j$ at stop $s$, the index $c$ refers to the different passengers groups, each of these with their own value of time. ${ }^{1}$

Constraints (4) ensure that overtaking does not occur. $\alpha_{i-1}^{S}$ is the departure time from stop s for bus $i-1$, while $H_{k, i-1, i}$ is the headway between busses $i-1$ and $i$ belonging to bus line $k$. Constraints (5) ensure that the departure time $\pi_{k}$ from the initial stop of the first bus on bus line $k$, is positive. Constraints (6) and (7) ensure that all $H_{k, i-1, i}$ and $\alpha_{i}^{S}$ are positive, while constraints (8) and (9), respectively, indicate that the dwell time $\beta_{i}^{S}$ of a bus $i$ at stop $s$ and, finally, the station-specific changing and orientation time $\delta^{s}$ equivalent to the minimum amount of time a passenger needs to change platform at stop s should be positive. This value is an input data applied to make more robust transfers and enhance the probability that passengers make the transfer even when services experience disruptions. Finally, the three different sets $K, M$ and $N$ refer to the set of bus lines, bus groups and transfer stops, respectively.

\footnotetext{
${ }^{1} c=1$ : Commuter trips (35.4 DKK/h). $c=2$ : Business trips $(270 \mathrm{DKK} / \mathrm{h}) . c=3$ : Leisure trips $(12.6 \mathrm{DKK} / \mathrm{h})$.
} 


\subsection{Heuristic solution approach}

This bi-level minimisation problem is extremely difficult to solve mathematically, since the timetable optimisation is a non-linear non-convex mixed integer problem (NP-Hard according to Nachtigall and Voget, 1996; Cevallos and Zhao, 2006), with passenger flows defined by the route choice model, where the route choice model is a non-linear non-continuous mapping of the timetable. Therefore, a heuristic solution approach was developed based on the idea of varying the offset of the bus lines. A variation in the offset value for a bus line affected the waiting time experienced at any transfer stop this bus passed.

Changing busses' offset value can be done at three levels: the most disaggregate, where the offset of every single bus is changed, a more aggregate level, where groups of busses (typically with similar characteristics) are changed together, and the line level, where every single bus belonging to a certain line are subject to an offset change. In this paper it was chosen that groups of busses from the same line were subject to offset changes. This choice was a compromise between optimisation potential (often larger when being at a more disaggregate level) and maintaining the existing structure of the timetable including typically fixed equal headway for each bus line for certain time intervals. Bus groups are created based on time of day and travel direction (e.g., in the evening period, roads are less congested, which implies that the average travel time is less than during peak hours). Due to the non-symmetric travel times (different in each direction), there is a group of busses for each direction of a bus line. Therefore, busses from a certain line have fixed headway in each direction and in each time period. The chosen approach allowed a potentially larger reduction in weighted transfer waiting time, while neither changing the headway of a bus line in the forward direction nor in the backward direction. However, the layover time might be changed. In theory this could have an impact on the fleet size. Including an evaluation of the impact on fleet size of every bus line's offset change was out of the scope of this article, but clearly a topic for future research.

In Fig. 1, an example of an offset change is indicated. The thin black arrows are one group of busses running in forward direction, the dashed arrows indicate the change in offset for that group of busses, while the thick black arrows represent a bus group from the same bus line running in the opposite direction.

A Tabu Search algorithm was applied to find the appropriate bus lines and their most promising offset changes. The reason for applying Tabu Search was because of its ability to search the solution space intelligently, i.e., to escape local minima and prevent cycling through the solution space (Glover, 1990).

\section{Time}

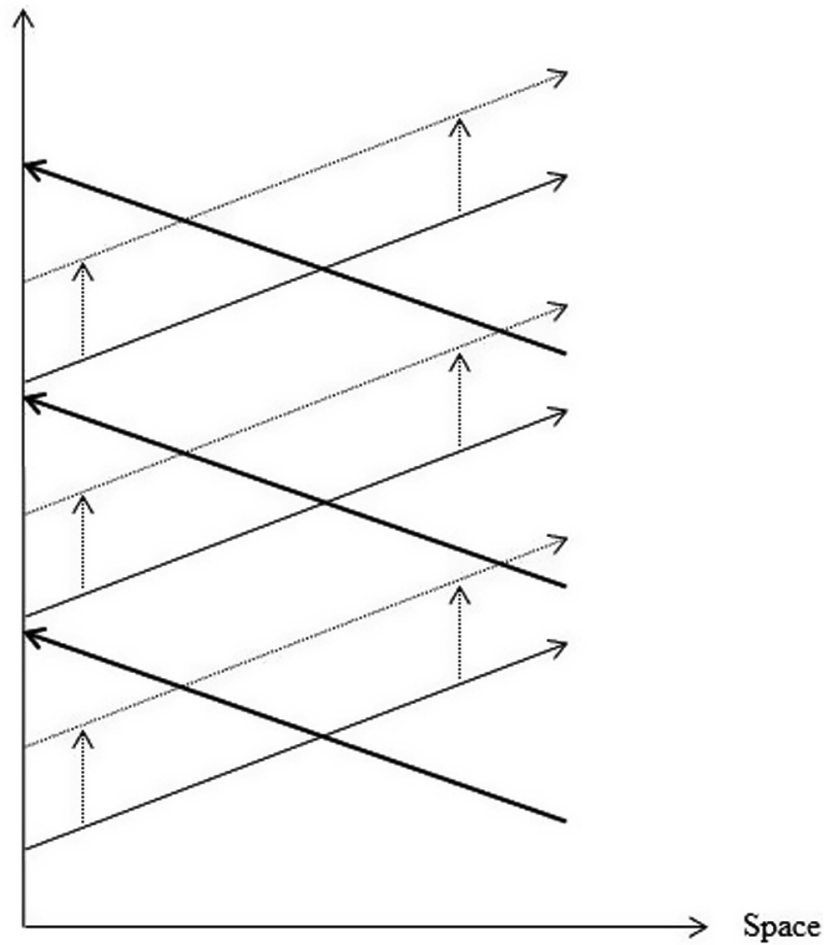

Stop

Fig. 1. Offset change. 


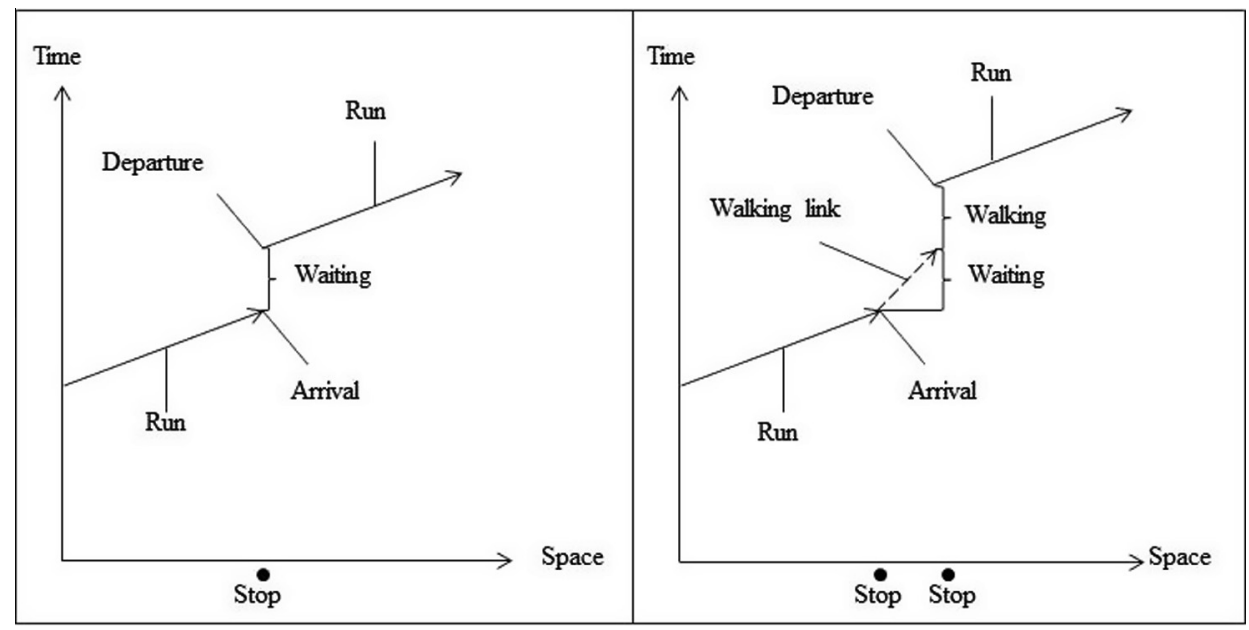

Fig. 2. (a) Direct transfer and (b) transfer including a walk.

The explicit consideration of passengers' modified route choice as a course of changes in the timetable in the present study was done as a sequential process, where the output of the timetable optimisation (i.e., the new timetable) served as input to the public assignment model. The output from this public assignment model (i.e., the passengers' travel patterns) was then used as input in the timetable optimisation. The process was continued until the objective value began to converge.

The entire heuristic solution approach worked according to the following step-wise approach elaborated in the following.

0. Run public assignment.

1. Calculate objective value.

2. Calculate optimisation potential for each offset change.

3. Impose offset changes.

4. If stopping criterion met, stop.

5. Otherwise, run public assignment and go to 1 .

\subsubsection{Calculation of objective value}

The aim of this optimisation was to minimise the overall weighted waiting time experienced by passengers when transferring in the transit network. After each public assignment calculation, the objective value was calculated. To do this properly, we needed to distinguish between direct transfers and transfers including walking.

Fig. 2(a) and (b) depicts a direct transfer and a transfer including a walk in a time-space diagram, respectively. From the figure it is evident that the waiting time is the time spent at the stop from which the next bus run departs. Calculating the weighted waiting time spent when transferring for the two types of transfers was done in the following two ways respectively according to Fig. 2, with the index $c$ referring to the trip purpose.

$$
\begin{aligned}
& \sum_{c}(\text { Departure }- \text { Arrival }) *\left(\text { VoT }_{c} * \# P a x_{c}\right) \\
& \sum_{c}(\text { Departure }- \text { Arrival }- \text { Walktime }) *\left(\operatorname{VoT}_{c} * \# \text { Pax }_{c}\right)
\end{aligned}
$$

\subsubsection{Optimisation potential}

The decision variables in this problem were the offset of the bus lines. Therefore, it was necessary to assess how changing the offset of a given bus line affected the solution value. This impact was treated as an estimate of the improving effect on the solution value of a given offset change for a given bus line and was referred to as optimisation potential. The reason for naming it potential was due to the uncertainty in the calculations (i.e., there might be a difference between the calculated optimisation potential and the realised improvement due to passengers' adapted behaviour). Ideally, the impact on the objective value for each offset change should be assessed by a public assignment calculation. But since this would be extremely timeconsuming, approximations were used instead.

To search the solution space comprehensively, a large neighbourhood needed to be considered. This was done by calculating the optimisation potential for every bus line (with at least two runs) in the interval between $\left[-h_{\max } ;+h_{\max }\right]^{2}$ with

\footnotetext{
${ }^{2} h_{\max }$ was the maximum headway for a given bus. If the maximum headway of a bus line was 5 min the optimisation potential was calculated for the following offset changes $(-5,-4,-3,-2,-1,0,1,2,3,4,5)$, where 0 was equal to the original offset.
} 


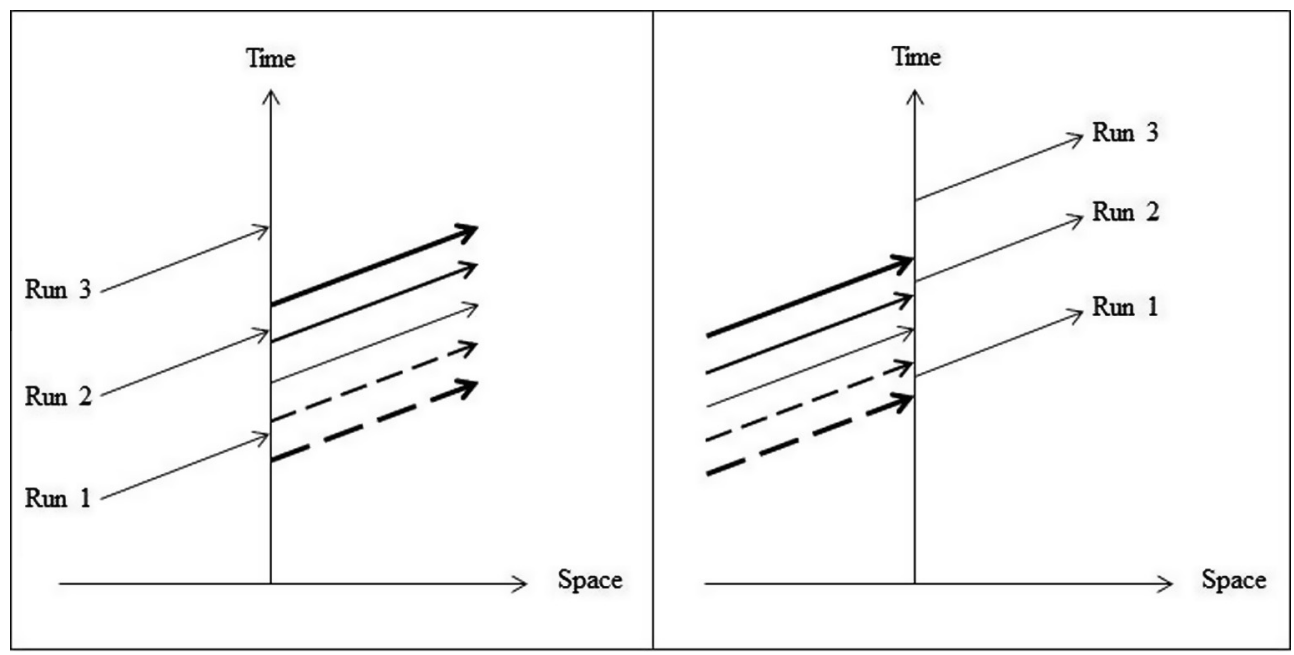

Fig. 3. (a) Feeding transfer and (b) connecting transfer.

increments of one minute, while maintaining the offset of all other bus lines. To ensure that all relevant passenger interactions were taken into account, and not just observed at a single transfer point, we distinguished between transfer points where passengers transfer either to (Fig. 3a) or from (Fig. 3b) the bus line of interest.

Travellers' transfer patterns were revealed from the public assignment calculation. Based on this, the impact of an offset change for a bus line was assessed under the assumption that passengers' transfer patterns were unaffected by offset changes. Considering a given bus line, the first task was to identify the transfer points where passengers transferred to or from the bus line. Having identified all the transfer points of a bus line enabled us to estimate the optimisation potential.

In Fig. 3, possible effects (single transfer point) of offset changes can be observed. Offset changes of the bus line of interest are marked by thicker (forward) and dashed (backward) arrows in a time-space diagram. In Fig. 3(a), a large forward offset change results in less transfer waiting time for passengers from run 2 . On the other hand, a large backward offset change means that people from run 1 are not able to board the bus line. In such cases the given offset change was penalised heavily to avoid a situation where predicting the passenger adaptions became impossible.

Calculating the optimisation potential for feeding transfers was done as outlined below (the approach used for connecting transfers was to a large extent similar and therefore omitted). The calculations were performed for each transfer point and for every feasible offset change of the current bus line according to the results from the public assignment calculation.

1. Sort runs of current bus line according to their departure times (ascending order).

\section{Direct transfer}

Calculate the waiting time between feeding run $i$ and run 1 (i.e., earliest departing run) of the current bus line at transfer station $s$ in the following way

$w_{i, 1}^{s}=\operatorname{dep}_{i}^{s}-\operatorname{arr}_{1}^{s}$

where $\operatorname{dep}_{i}^{s}$ is the time at which run $i$ departs from station $s$, and $\operatorname{arr}_{1}^{s}$ is the time at which run 1 arrives at station $s$.

If $w_{i, 1}^{S}<0$, then select the second earliest departing run of the current bus line and calculate $w_{i 2}^{S}$. The process continues until $w_{i, j}^{S}$ turns positive or equals zero for a given $j$ or until all runs of the current bus line are examined. In the latter case, penalise $w_{i j}^{S}$ to avoid unpredictable offset changes.

3. Transfer including walk

Calculate the waiting time between feeding run $i$ and run 1 of the current bus line at transfer station $s$ in the following way

$w_{i, 1}^{s}=\operatorname{dep}_{i}^{s}-\left(\operatorname{arr}_{1}^{s 1}+\right.$ walk $\left.^{s, s 1}\right)$

The parameter walk ${ }^{s, s 1}$ is the time it takes to walk from station $s$ to station $s 1$.

If $w_{i, 1}^{S}<0$, then select the second earliest departure and calculate $w_{i, 2}^{S}$ The process continues until $w_{i, j}^{S}$ turns positive or equals zero for a given $j$ or until all runs of the current bus line are examined. In the latter case, penalise $w_{i, j}^{S}$.

4. Multiply $w_{i, j}^{S}$ by the weight factor (number of passengers and their value of time) for the particular transfer.

5. The optimisation potential for the specific offset change is now equal to the difference between the value calculated in step 4 and the product of $w_{i, j}^{S}$ and $\omega_{i, j}^{S}$ calculated according to the do-nothing scenario (i.e., where offsets are not changed).

This process was repeated until both connecting and feeding transfers for all bus lines had been examined. 


\subsubsection{Imposing offset changes}

Having calculated the optimisation potential of every feasible offset change of all bus lines, the next step was to impose a subset of these. Examining the optimisation potential of every offset change enabled us to impose the offset change with the largest optimisation potential, under the condition that the current bus line was not labelled as tabu. After imposing the most promising offset change, the bus line was labelled as tabu. We also prohibited offset changes on bus lines comprised in the sub-network of the current bus line (see Section 2.2.4).

This process continued until no offset changes could be imposed without violating the sub-network constraint, and no positive optimisation potentials existed for any bus lines not labelled as tabu. The reason for labelling a bus line as tabu rather than only labelling a certain offset change as tabu was to ensure sufficient diversification, when exploring the solution space.

\subsubsection{Sub-networks}

Performing several offset changes based on optimisation potentials without calculating their exact impact from a public assignment calculation was based on dividing the transit system into sub-networks with no or only negligible passenger interaction. Sub-networks were not predefined and static, but simply created on the go when offset changes were imposed. Every bus line had its own sub-network comprising all bus lines crossing its trajectory and bus lines with passenger interaction (either direct or by a walking link). This meant that the order in which bus lines were chosen affected the way in which sub-networks were formed, hence prohibiting certain bus lines to having their offset changed. After imposing an offset change on a bus line, we prohibited offset changes on bus lines comprised in this bus line's sub-network. Simply because changing the offset of two bus lines with significant passenger interaction could have a counteracting effect on the total waiting time.

The assumption about no passenger interaction between different sub-networks was legitimate, when journeys in the transit system only consisted of either one or two trips (e.g., Bus or Bus-> Train). This assumption was important to impose offset changes on more than one bus line before running another transit assignment, given the large calculation time of the assignment model. However, the output data did not reveal passengers' exact route choice, only transfer patterns were revealed, not the entire journey. Therefore, only services with direct passenger interaction were identified from the output data. From the Danish national transport survey, we know that only $5 \%$ of all transit journeys consist of three or more trips (DTU Transport, 2013). If the second leg in a three leg journey was performed by bus (around $1 \%$ in total according to DTU Transport (2013)), first and last legs were comprised in the sub-network. Hence, only in around $4 \%$ of all transit journeys, a part of the passenger interaction was left unrevealed when applying the described methodology. Consequently, the optimisation potential estimated for every offset change should be close to the one revealed from the transit assignment.

When applying this methodology to other transit networks, it is essential to have knowledge about the amount of journeys consisting of 3 or more legs. The higher the share of long-chained trips, the less certain the estimated optimisation potentials become. Therefore, under particular severe circumstances (e.g., networks where the majority of journeys are long-chained) it can be necessary to assess the optimisation potential with a transit assignment calculation. However, developing smarter strategies might be a first step e.g., creating larger sub-networks.

\subsubsection{Stopping criterion}

The process of imposing potential improving offset changes (upper level) continued until no feasible and improving offset changes remained. Then another public assignment calculation (lower level) was run to reveal the adapted passenger behaviour. After a public assignment calculation, all non-tabu bus lines were again subject to offset changes. The entire process (upper and lower levels) continued until the objective value converged.

\subsubsection{Pseudo-code}

The following pseudo-code gathers the threads from the previous sub-sections and presents the entire algorithm in a clearer way.

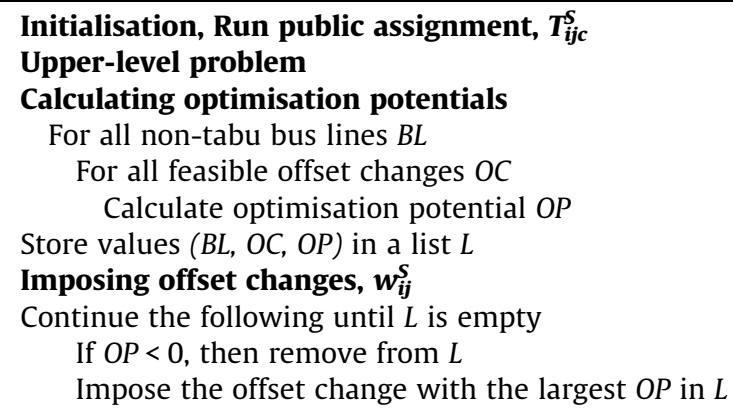


Label $B L$ as tabu

Derive sub-network sn for $B L$

Remove all values sn from $L$

Go to Lower-level problem

Lower-level Problem

Run public assignment, $\boldsymbol{T}_{\text {ijc }}^{\boldsymbol{S}}$

Calculate solution value

If stopping criterion is met, terminate.

Otherwise, go to upper-level.

The initialisation yielded passengers' travel behaviour. The relevant information in this context was how many passengers $T_{i j c}^{S}$ were transferring between services at which stations. Recall that $T_{i j c}^{S}$ was the number of passenger transferring from bus line $i$ to bus line $j$ at transfer stop $s$, while $c$ referred to the different passenger groups. The minimisation problem was a bi-level minimisation problem, where the upper-level problem was the timetable optimisation. Based on the number of passengers transferring, $T_{i j c}^{S}$, calculated in the lower-level problem, bus lines' offset values were changed to optimise transfer waiting time, $w_{i j}^{S}$. The modified offset values served as input (together with the other network characteristics) for the lower-level problem, where passenger flows were derived by a route choice model. The lower level problem yielded the number of passengers transferring between two lines at a certain stop, $T_{i j c}^{S}$. This sequential bi-level optimisation process continued until the stopping criterion was met.

\section{Data}

The described optimisation approach was tested on the public transit network in Denmark on the basis of the newly developed Danish National Transport Model. This model is currently under development and the final version (2.0) is scheduled for 2015. The version used for public assignment calculations in this study was version 1.0. In this section the model and the bus network in Denmark are described.

\subsection{Public assignment model}

The public assignment model was schedule-based, which meant that every single run of the bus lines was described. Demand was assigned uniformly within 10 different time-of-day periods. The model applied a utility-based approach to describe travellers' perceived travel costs. The formulation of the utility function reflected the perceived cost of travelling from zone $i$ to zone $j$ at time $t$ for passenger group $c$ (i.e., generalised travel cost) as follows.

$$
\begin{aligned}
C_{i j t c}= & \beta_{c} * \text { WaitingTime }_{i j}+\beta_{c} * \text { WaitInZoneTime }_{i j}+\beta_{c} * \text { WalkTime }_{i j}+\beta_{c} * \text { ConnectorTime }_{i j} \\
& +\beta_{c} * \text { NumberOfChanges }_{i j}+\beta_{c} * \text { TotalInVehicleTime }_{i j}, \forall t, c
\end{aligned}
$$

In this formula, $C_{i j t c}$ is the utility, WaitingTime is the transfer waiting time, WaitInZoneTime is the waiting time at home or in the origin zone, WalkTime is the walking time used when transferring, ConnectorTime is the time used for getting from home to the desired transit station, NumberOfChanges is the number of transfers during a journey, and TotalInVehicleTime is the time spent driving in transit vehicles. Together these parameters reflect each traveller's disutility associated with a trip in the transit system. The $\beta$ 's represent the weights of each of the 6 parameters. For each passenger group the beta values are outlined in Table 1. All beta values except the ones for ChangePenalty are in DKK/minute. ChangePenalty is an impedance cost incurred for every transfer. ${ }^{3}$ Based on this it is easy to tell that transit users are assumed to be transfer averse e.g., commuters prefer 4 min extra travel time to a journey including a transfer.

The transit fare system in Denmark is mostly OD-based, hence to a large extent independent of passengers' route choice. It was thus not the level of VoT as such that influence passengers' route choice but rather the ratio between the different time components. The fact that business travellers had significantly large time values compared to commuter trips and leisure trips did not bias the optimisation, since business trips only comprised $2.3 \%$ of all transit trips.

Travellers' route choice behaviour was based on utility maximisation and the travellers were assumed to have complete knowledge of the entire network and timetables. Albeit this assumption seems optimistic, passenger information has reached a level with real-time information available on webpages, cell phone-apps and stations, which implies that the assumption in many ways is realistic. Theoretically, this means that passenger flows derived from SUE and UE become similar. Deriving the SUE of a transit network, passengers optimise their perceived utility from their known set of paths from origin to destination. In the UE, passengers are assumed to be familiar with all paths, and choose the one that maximise their utility. Providing the passengers with sufficient real time information on the state of the transit system, the UE and SUE coincide since the perceived utility become equivalent to the objective utility.

\footnotetext{
${ }^{3}$ The values build on the critical study of Nielsen (2000), but were recalibrated in the Danish national transport model to fit the passenger flows to observed counts.
} 
Table 1

Beta values (VoT) and share of trips.

\begin{tabular}{lllllccc}
\hline Trip types & WalkTime & WaitingTime & ConnectorTime & WaitInZone & ChangePenalty & BusInVehicleTime & Share of all trips (\%) \\
\hline Commuter & 0.633 & 0.59 & 0.64 & 0.28 & 2.20 & 0.56 \\
Business & 4.50 & 4.50 & 4.50 & 2.35 & 18.8 & 4.70 \\
Leisure & 0.209 & 0.21 & 0.21 & 0.117 & 1.10 & 0.19 & 55.4 \\
\hline
\end{tabular}

The network loading was done by an all-or-nothing assignment where all passengers were loaded onto the routes that maximised their utility. It could be argued that it would have been more realistic if the stochastic user equilibrium was found instead. However, due to uniformly distribution of departure time, different routes might have been used between each ODpair at different departure times during the day. Likewise, it is extremely seldom that passengers in the Danish transit network are rejected entering coaches due to crowding, and most passengers get seats while on-board. A pure user equilibrium method would hence resemble an all-or-nothing model with very few exceptions. For the same reason vehicle capacity (i.e., also the ability to board a transit vehicle) is not modelled in the Danish national transport model. However, crowding could be built into the route choice model with a flow-dependent cost function. The passenger flows that were optimised would hence depend on the crowding function.

The method developed in the current paper was a bi-level optimisation between the timetable optimisation resulting in a better synchronisation and a route choice model deriving passengers' travel behaviour. A better synchronisation would generally yield benefits independent on crowding. For example if 100 passengers transferred from a low frequent train at time 00 to a bus service with 10 min frequency and a capacity per bus of 80 passengers. If the prior bus schedule ran at minutes 08 and 18 , then 80 passengers had to wait $8 \mathrm{~min}$ and 20 had to wait $18 \mathrm{~min}$. If the optimised schedule was 01 and 11 , then both groups of passengers would gain 7 min of transfer time.

It is true though, that the political constraint that all schedules should keep their frequency - e.g., 10 min - may be less well in the crowded case than the uncrowded, e.g., in the example above one may run two busses at 01. This is a trickier problem though, because then you will also get a lower frequency along the route, hence more waiting time for the nontransferring passengers who arrive at the station at random. It is trivial to relax the headway restriction. If the network is recoded so each departure has its own line number, and a crowding function introduced, then all departures will be optimised independent of each other, and the crowding case mentioned above be solved. The optimisation would presumably result in larger reductions of the KPIs introduced in Section 4. But the structure and memorability of the timetable will be lost, and at the same time the requirements for rolling stock may also increase. Finally, passengers arriving at their station at random will be affected by longer waiting times due to busses bunching up.

\subsection{The public transport network in Denmark}

In Fig. 4 all transit lines in Denmark are outlined. This figure shows that the transit network in Denmark consists of several smaller networks in the larger cities and a fair amount of regional and inter-city lines connecting these. The network consists of the following:

- 1794 public lines (e.g., train, bus, metro, S-train etc.), of which 1440 are busses.

- 8373 variants of the public lines, of which 7877 are busses.

- 22,187 stops, of which 21,396 are bus-stops.

- 1077 zones, 3 trip purposes and hence app. 3.5 million OD cells.

Since train schedules have many restrictions - e.g., limited overtaking possibilities at double-track lines and fixed meeting stations at single-track lines - it was decided to assume that train schedules were fixed and only bus schedules were subject to offset changes. Passengers transferring to/from other modes than busses were considered as well.

O/D-matrices for 10 different time intervals representing a single day were used to describe the demand. Within each time interval, travellers were split uniformly into 2-min intervals and launched within each of these. In the test of the approach, only evening period from 6 pm to 9 pm was considered since most transit lines ran with lower frequency, potentially leaving a larger potential for improvement.

In Denmark, there are some provincial towns with minor bus networks. However, even in more provincial parts of the network, there are regional busses that connect towns, and it is hence difficult to extract sub-networks. Therefore, it was chosen to consider the entire bus network of Denmark as subject to the timetable optimisation. An important point for considering the entire bus network was also that the algorithm was designed to optimise under variable demands, and did not change interdependent bus lines at the same iteration. Instead, the most promising ones were modified while all crossing bus lines were locked (see Section 2.2.4). Afterwards, passenger flows were recalculated with the route choice model and based on these, the objective value was updated. If all bus-routes (including dependent bus lines) were optimised in a sub-network at the same time in the inner loop, there was the risk, that flows and hence also the objective function in the next iteration deviated too much from the flows in the previous iteration, and that the algorithm thus would oscillate 


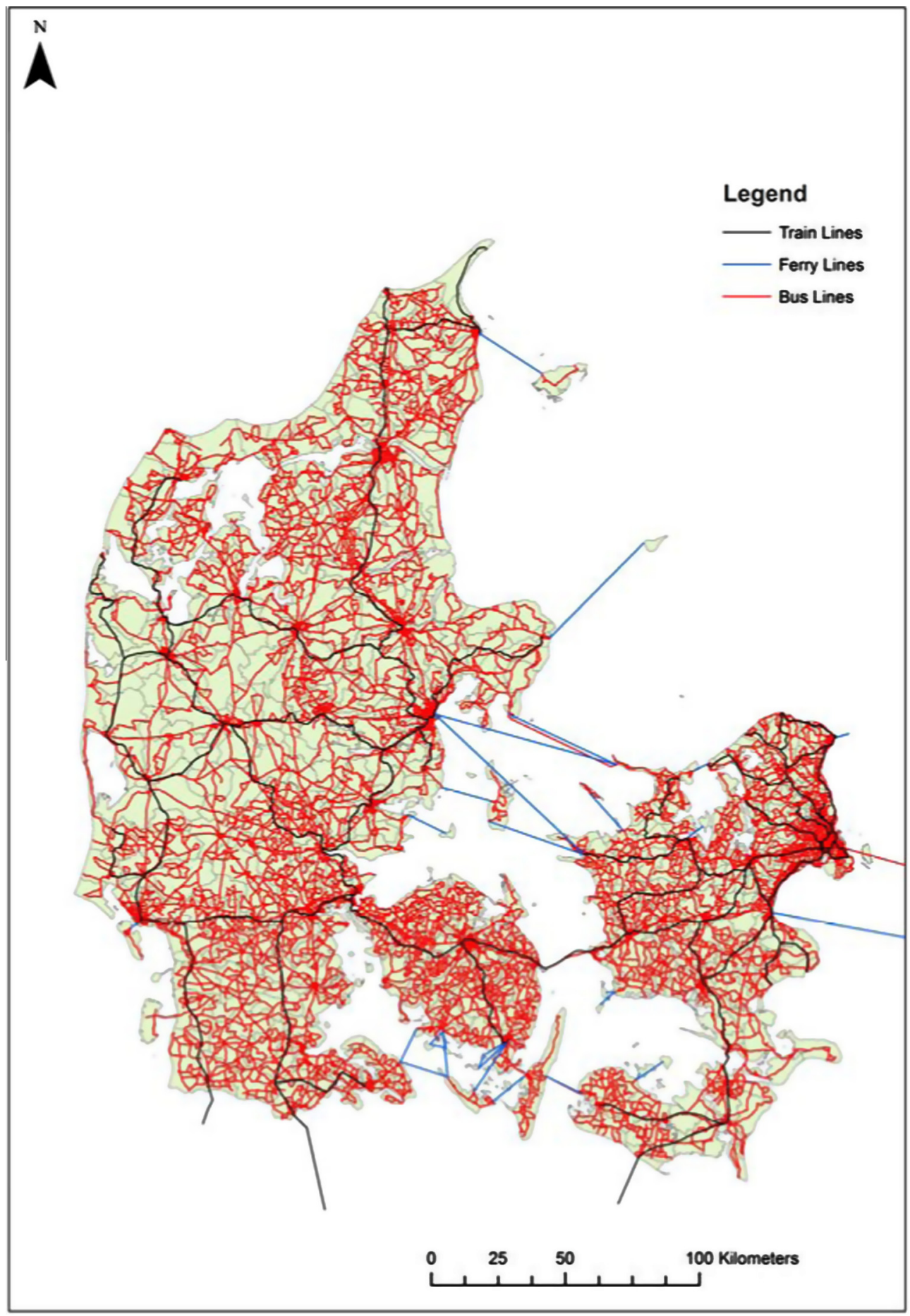

Fig. 4. Transit lines in Denmark.

and converge slower. A key assumption in the algorithm was thus, that the candidate bus line was solved to optimality given the flows from the prior iteration. This made this bus line more attractive and it meant that the flows on this bus line would change (mainly increase, but also shift between departures). The transfer patterns to all crossing bus lines would then change, but how they actually changed was first revealed when the route choice model was run in the next iteration of outer bi-level problem. 
To get an impression of the complexity of the bus network, Fig. 5 shows an example of bus lines (turquoise) that crosses bus line 200S in Copenhagen. It is clear that there are many interdependencies in the network, and if these bus lines were to be optimised on flows that were too far from the timetable in the present iteration, the algorithm would never converge.

\section{Results and discussion}

This section presents the results obtained when applying the heuristic solution approach to timetable optimisation to the Danish public transport network. It should be noted that the network contained transit lines from all over Denmark, but only bus lines were subject to offset changes. Table 2 shows that it took 5 iterations before the bi-level timetable optimisation converged. At this point, the weighted waiting time was reduced by more than $5 \%$, while barely affecting the other time components of the journeys. Fig. 6 illustrates that the largest improvement in objective value occurred over the first couple of iterations, while the latter iterations showed that the objective value converged.

The generalised travel cost was reduced by $0.3 \%$, which meant that transit users in general were better off than before the optimisation. By showing this, it was proven that the weighted transfer waiting time was not just reduced to the detriment

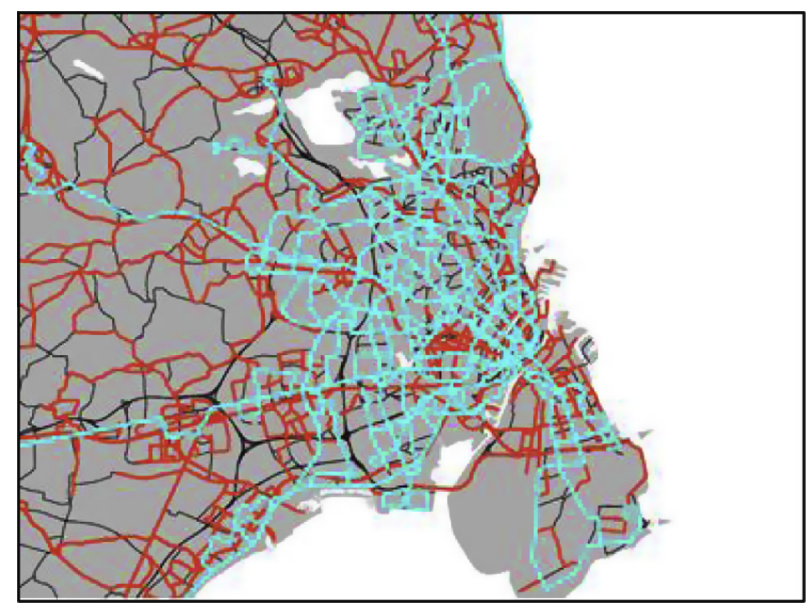

Fig. 5. Transit lines crossing bus lines 200S.

Table 2

Development in solution value.

\begin{tabular}{|c|c|c|c|c|}
\hline & \multicolumn{4}{|l|}{ 0th iteration } \\
\hline & Generalised travel cost & Waiting time & Walking time & In-vehicle time \\
\hline Total (DKK) & 6320322.78 & 125777.54 & 46925.99 & 1598292.26 \\
\hline $\begin{array}{l}\text { Total (DKK) } \\
\text { Absolute change } \\
\text { Percentage change }\end{array}$ & $\begin{array}{l}6314552.19 \\
5770.59 \\
-0.09\end{array}$ & $\begin{array}{l}\text { 1st iteration } \\
121932.69 \\
3844.86 \\
-3.06\end{array}$ & $\begin{array}{l}47071.99 \\
-146.00 \\
0.31\end{array}$ & $\begin{array}{l}1599006.58 \\
-714.32 \\
0.04\end{array}$ \\
\hline $\begin{array}{l}\text { Total (DKK) } \\
\text { Absolute change } \\
\text { Percentage change }\end{array}$ & $\begin{array}{l}6312161.07 \\
8161.71 \\
-0.13\end{array}$ & $\begin{array}{l}\text { 2nd iteration } \\
119527.52 \\
6250.02 \\
-4.97\end{array}$ & $\begin{array}{l}47001.78 \\
-75.79 \\
0.16\end{array}$ & $\begin{array}{l}1599357.19 \\
-1064.92 \\
0.07\end{array}$ \\
\hline $\begin{array}{l}\text { Total (DKK) } \\
\text { Absolute change } \\
\text { Percentage change }\end{array}$ & $\begin{array}{l}6301220.29 \\
19102.49 \\
-0.30\end{array}$ & $\begin{array}{l}\text { 3rd iteration } \\
119311.98 \\
6465.56 \\
-5.14\end{array}$ & $\begin{array}{l}47297.51 \\
-371.52 \\
0.79\end{array}$ & $\begin{array}{l}1599200.96 \\
-908.70 \\
0.06\end{array}$ \\
\hline $\begin{array}{l}\text { Total (DKK) } \\
\text { Absolute change } \\
\text { Percentage change }\end{array}$ & $\begin{array}{l}6302028.98 \\
18293.80 \\
-0.29\end{array}$ & $\begin{array}{l}\text { 4th iteration } \\
119369.32 \\
6408.22 \\
-5.09\end{array}$ & $\begin{array}{l}47264.52 \\
-338.53 \\
0.72\end{array}$ & $\begin{array}{l}1599175.41 \\
-883.15 \\
0.06\end{array}$ \\
\hline $\begin{array}{l}\text { Total (DKK) } \\
\text { Absolute change } \\
\text { Percentage change }\end{array}$ & $\begin{array}{l}6302235.64 \\
18087.14 \\
-0.29\end{array}$ & $\begin{array}{l}\text { 5th iteration } \\
119394.24 \\
6383.30 \\
-5.08\end{array}$ & $\begin{array}{l}47235.01 \\
-309.02 \\
0.66\end{array}$ & $\begin{array}{l}1599243.69 \\
-951.43 \\
0.06\end{array}$ \\
\hline
\end{tabular}




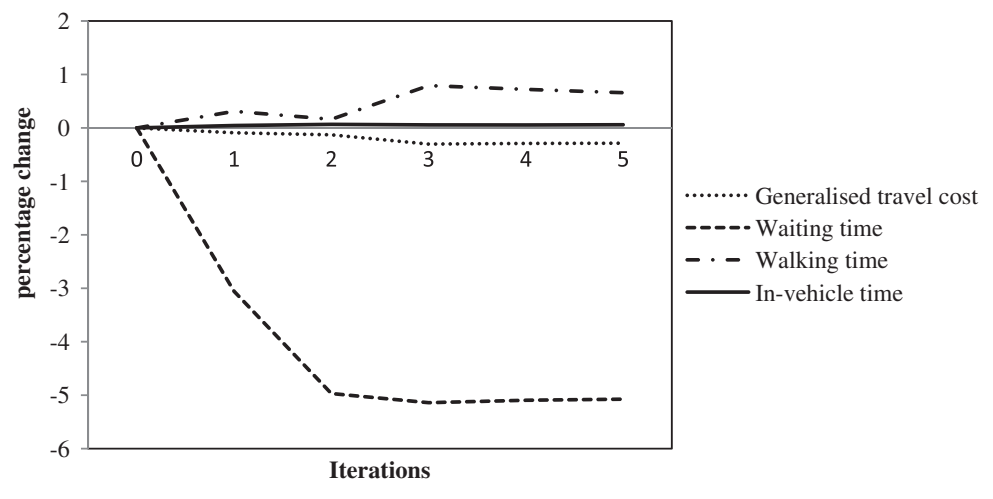

Fig. 6. Development in solution value.

of something else. The generalised travel cost would reveal if offset changes implied that busses started to bunch up. In that case passengers would have to wait longer at their boarding stops and also at some non-prioritised transfer stops. In the current study, bus bunching was prevented by only allowing offset changes to be imposed on entire groups of busses at the time.

Walking time increased by $0.66 \%$ which was explained by the increase in attractiveness of the transfer possibilities. Therefore, users in the transit system might have preferred to add an extra transfer to get from A to B, even though it included some walking. From Table 1 it is evident that beta values for walking time and waiting are almost equal. This could be interpreted as passengers, who have chosen to make a transfer, were indifferent between waiting and walking. Likewise, it was seen that these beta values were close to the ones for in-vehicle travel time. Therefore, it was reasonable to assume that Danish transit users were more focused on the total travel time from origin to destination rather than whether they were walking, waiting or being in a transit vehicle.

The small increase in in-vehicle time could be a result of a minor change in the users' travel patterns due to offset changes as well as passengers shifting from long access mode (walk/bicycle) to stations to bus, if the bus was better coordinated with the train service.

Despite exhibiting monetary reductions in the different time components as outlined in Table 2, the monetary reduction is not equivalent to an increase in revenue for the bus company. It should rather be seen as what passengers are willing to pay to avoid excessive waiting time, e.g., as evaluated in socio-economic cost benefit analyses (CBA). Since bus companies' operations are subsidised by the public authorities in Denmark (government, regions, municipalities), the CBA is indeed a criterion when deciding upon the subsidy.

Examining benefits at the zone level exhibited in Fig. 7 enabled three different conclusions to be drawn. For the four largest cities in Denmark (in terms of population) it was seen that the zones around Aalborg and Copenhagen experienced less waiting time reduction than the zones around Aarhus and Odense. This was explained by the great focus on coordination of public transport in Copenhagen and Aalborg the recent years and a strategy of less bus lines with a high frequency, so called "A-busses" or "metro-busses", respectively. However, it was encouraging to see that the applied methodology yielded an even further reduction in waiting time (better coordination). On the western part of the Zealand a lot of zones experienced a significant reduction of weighted waiting time. In this part of Zealand, the old county planned bus lines independently of the railway operations. For that reason no coordination between the two modes were planned. Furthermore, the county did not collect detailed counts and thus did not systematically evaluate flows. Therefore, transit users in these zones benefited great from such a bus timetable optimisation. A somewhat similar pattern was seen on the island of Funen around the city of Odense. The transit planning on Funen used to be split between the 32 different municipalities, which explained the lack of overall coordination compared e.g., to Copenhagen, where the public company Movia (prior HUR, and HT) organise and tender the bus operations on behalf of all municipalities. It should be noted that a recent public sector governance reform has extended Movia's responsibility to the whole Zealand and made a similar organisation for Funen, but this has not yet led to major changes of the timetabling yet.

The developed timetable optimisation approach was also applied to the transit network in the Greater Copenhagen area, where the timetable used during the morning peak hours served as initial solution. The change in weighted waiting time at the zone level is shown in Fig. 8, where it is seen that primarily zones lying along the train lines experience a better coordination of transit services. This was because of the large passenger flows at train stations (e.g., from bus to train or the opposite way around). In the Greater Copenhagen area train stations were often used as bus hubs, which meant that several bus lines served as feeder modes and connecting modes for the trains here. Despite the reduction during the morning peak hours for the Greater Copenhagen area, it was not possible to obtain the same large magnitude of weighted waiting time reduction as for the evening period. This was explained from the higher frequency during rush hours compared to the evening period examined in the national transport model (see Fig. 6). 


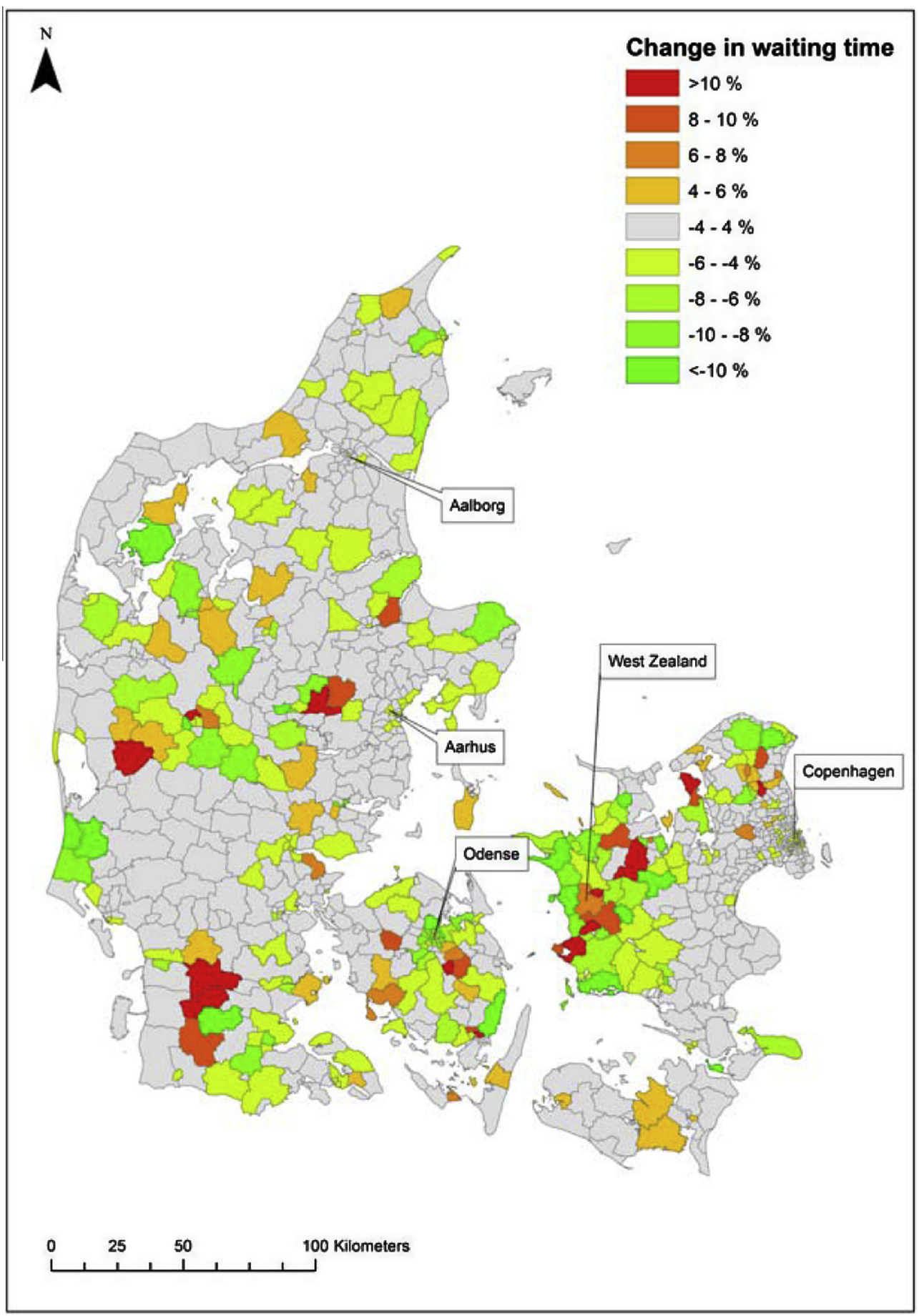

Fig. 7. Waiting time change (percentage) at zone level (Denmark).

The improvement of the generalised cost adds up to approximately 45 million DKK of value of time gains on a yearly basis (if summarised to a full day of operations and a full year), which is a large benefit for the society, given that it entirely comes from optimisation of the timetabling at limited extra costs. It is important to be aware that the reduction in weighted transfer waiting time that the present optimisation yielded is not equivalent to increased revenue for the bus company. Assigning monetary values to waiting time should rather be seen as an indication of how much passengers are willing to pay to avoid this extra waiting time. However, when travelling by bus becomes more attractive from a user's perspective, bus as travel mode may experience an increase in market share, hence providing larger revenue. Fortunately, an improvement in transfer 


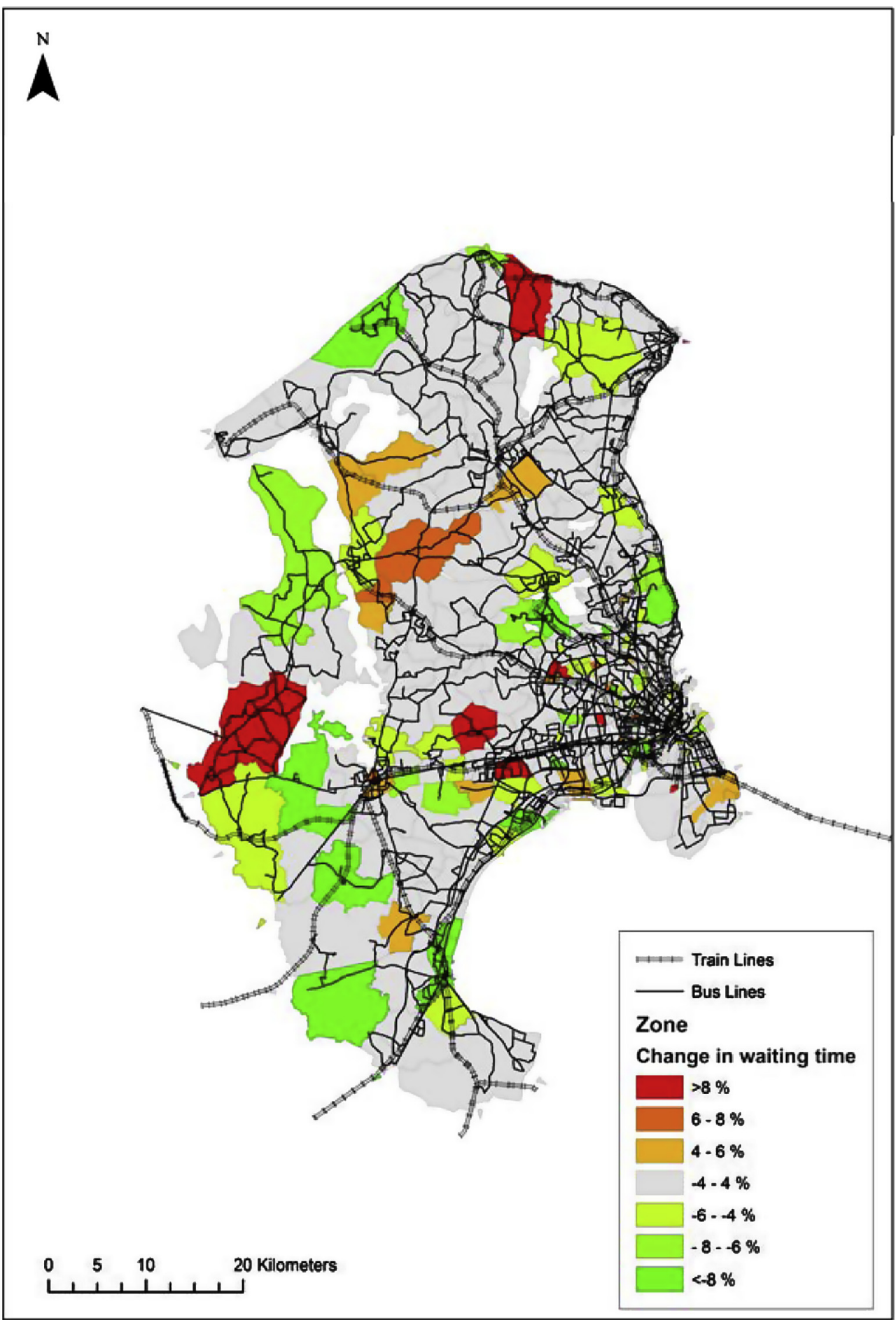

Fig. 8. Waiting time change (percentage) at zone level (Greater Copenhagen area).

waiting time as outlined in this paper can be obtained at a negligible cost. In this regard it should be mentioned that there is a gap between research and practice. Despite the promising results outlined, the bus company may have several other reasons to consider changing the timetable inappropriate.

It is important to acknowledge the deviating desires among the different stakeholders. According to one of the Danish bus operators, Movia, the fleet size required to fulfil the contractual obligations is, because of the high expenses, the main 
priority for bus companies. Customers, on the other hand, are mainly interested in a fast and reliable service between origin and destination (Ceder, 2007). Therefore, in the case a transfer is needed to complete a journey in the transit system, passengers prefer if the system is coordinated in such a way that waiting time is minimised. The two different desires may not always go hand in hand as minimising transfer waiting time can cause an extra need for rolling stock, while minimising the fleet size can imply longer waiting time when transferring for the timetable to be feasible.

Regarding the gap between research and practice one way of improving the real-life applicability could be to assume stochastic travel times rather than deterministic travel times as in this study. Nuzzolo et al. (2012) applied a doubly dynamic schedule-based transit assignment taking into account how frequent transit users, based on a learning process, adapted their departure time, boarding stop choice and run choice. In this way, the results would be more robust towards deviations from the scheduled travel times and presumably more similar to real life. However, this would be extremely demanding in terms of computation time with such a large network as the Danish (see specifications in Section 3.2). In this case, also estimates of average delays should be known and extra buffer time in the transfer times should, if necessary, be imposed. Regarding the current model, this is not a major change, but when delay data is available only minor changes in the calculation of transfer times should be made. Nevertheless, this study should be seen as a proof of concept for combining optimisation methods and assignment models in transit systems. Potential extra features would be a topic for future research.

\section{Conclusion and future work}

The current study proposed a timetable optimisation approach that explicitly considered passengers' modified route choice as a reaction to changes in the timetable. The approach was applied to a real large-scale transit network. The optimisation yielded a significant reduction in weighted transfer waiting time, while only affecting the in-vehicle travel time and the generalised travel journey cost to a lesser extent.

Overall, the study contributes to the literature by proposing a new optimisation tool, which has its strengths with respect to both the timetable optimisation and the reliability perspective, since changes in the travellers' route choice decisions are considered explicitly whereby demand effects are taken into account.

A topic for future research could be to use stochastic rather than deterministic travel times. Another additional feature could be to consider the problem as multi-objective, e.g., minimise fleet size and transfer waiting time. In this way, convincing bus companies of the applicability of the results might be a smoother process. Finally, the order in which offset changes were imposed could be changed from the "greedy" approach introduced in this paper, where the feasible offset change with the largest optimisation potential was chosen. Instead, a knapsack-inspired approach could be applied. Combining the bus lines (for which the offset should be changed) in a way that yields the largest total potential improvement, while still obeying the idea of sub-networks, could be an idea for future research.

\section{References}

Bielli, M., Caramia, M., Carotenuto, P., 2002. Genetic algorithms in bus network optimization. Transp. Res. Part C Emerg. Technol. 10 (1), 19-34.

Bookbinder, J.H., Désilets, A., 1992. Transfer optimization in a transit network. Transp. Sci. 26 (2), 106-118.

Ceder, A., 2007. Public Transit Planning and Operation: Theory, Modeling and Practice. Elsevier, Butterworth-Heinemann.

Ceder, A.A., 2011. Public-transport vehicle scheduling with multi vehicle type. Transp. Res. Part C Emerg. Technol. 19 (3), $485-497$.

Cevallos, F., Zhao, F., 2006. Minimizing transfer times in public transit network with genetic algorithm. Transp. Res. Rec. J. Transp. Res. Board 1971 (1), $74-$ 79.

Constantin, I., Florian, M., 1995. Optimizing frequencies in a transit network: a nonlinear bi-level programming approach. Int. Trans. Oper. Res. 2 (2), 149164.

de Palma, A., Lindsey, R., 2001. Optimal timetables for public transportation. Transp. Res. Part B Method. 35 (8), 789-813.

DTU Transport, 2013. Transportvaneundersøgelsen 2006-2012.

Fan, W., Machemehl, R.B., 2006. Optimal transit route network design problem with variable transit demand: genetic algorithm approach. J. Transp. Eng. $132(1), 40-51$.

Feil, M., 2005. Optimisation of Public Transport Timetables with respect to Transfer (Master thesis).

Glover, F., 1990. Tabu search: a tutorial. Interfaces 20 (4), 74-94.

Guihaire, V., Hao, J.K., 2008. Transit network design and scheduling: a global review. Transp. Res. Part A Policy Pract. 42 (10), $1251-1273$.

Guihaire, V., Hao, J.K., 2010. Improving timetable quality in scheduled transit networks. Trends in Applied Intelligent Systems. Springer Berlin Heidelberg, pp. 21-30.

Hadas, Y., Ceder, A.A., 2010. Optimal coordination of public-transit vehicles using operational tactics examined by simulation. Transp. Res. Part C Emerg. Technol. 18 (6), 879-895.

Ibarra-Rojas, O.J., Rios-Solis, Y.A., 2012. Synchronization of bus timetabling. Transp. Res. Part B Method. 46 (5), 599-614.

Jansen, Leise Neel, Pedersen, Michael Berliner, Nielsen, Otto Anker, 2002. Minimizing passenger transfer times in public transport timetables. In: 7th Conference of the Hong Kong Society for Transportation Studies, Transportation in the information age. Proceedings, 14 December, Hong Kong, pp. 229239.

Kepaptsoglou, K., Karlaftis, M., 2009. Transit route network design problem: review. J. Transp. Eng. 135 (8), 491-505.

Knoppers, P., Muller, T., 1995. Optimized transfer opportunities in public transport. Transp. Sci. 29 (1), 101-105.

Lee, Y.J., Vuchic, V.R., 2005. Transit network design with variable demand. J. Transp. Eng. 131 (1), 1-10.

Liu, Z., Shen, J., Wang, H., Yang, W., 2007. Regional bus timetabling model with synchronization. J. Transp. Syst. Eng. Inf. Technol. 7 (2), $109-112$.

Ma, T.Y., 2011. A hybrid multiagent learning algorithm for solving the dynamic simulation-based continuous transit network design problem. In: Technologies and Applications of Artificial Intelligence (TAAI), 2011 International Conference on. IEEE, pp. 113-118.

Nachtigall, K., Voget, S., 1996. A genetic algorithm approach to periodic railway synchronization. Comput. Oper. Res. 23 (5), $453-463$.

Nielsen, O.A., 2000. A stochastic traffic assignment model considering differences in passengers utility functions. Transp. Res. Part B Method. 34B (5), 337402, Elsevier Science Ltd. 
Niu, H., Zhou, X., 2013. Optimizing urban rail timetable under time-dependent demand and oversaturated conditions. Transp. Res. Part C Emerg. Technol. $36,212-230$

Nuzzolo, A., Crisalli, U., Rosati, L., 2012. A schedule-based assignment model with explicit capacity constraints for congested transit networks. Transp. Res. Part C Emerg. Technol. 20 (1), 16-33.

Petersen, H.L., Larsen, A., Madsen, O.B., Petersen, B., Ropke, S., 2012. The simultaneous vehicle scheduling and passenger service problem. Transport. Sci. 47 (4), 603-616.

RH, 2009. Foer biltrafikken staar stille Hvad kan den kollektive transport bidrage med? Region Hovedstaden, Report. June 2009.

Wang, J.Y., Lin, C.M., 2010. Mass transit route network design using genetic algorithm. J. Chin. Inst. Eng. 33 (2), 301-315.

Wong, R.C., Yuen, T.W., Fung, K.W., Leung, J.M., 2008. Optimizing timetable synchronization for rail mass transit. Transp. Sci. 42 (1), $57-69$.

Yan, Y., Meng, Q., Wang, S., Guo, X., 2012. Robust optimization model of schedule design for a fixed bus route. Transp. Res. Part C Emerg. Technol. 25, 113121.

Yu, B., Yang, Z., Yao, J., 2009. Genetic algorithm for bus frequency optimization. J. Transp. Eng. 136 (6), 576-583.

Zhao, F., Ubaka, I., 2004. Transit network optimization-minimizing transfers and optimizing route directness. J. Public Transp. 7 (1), $63-82$. 